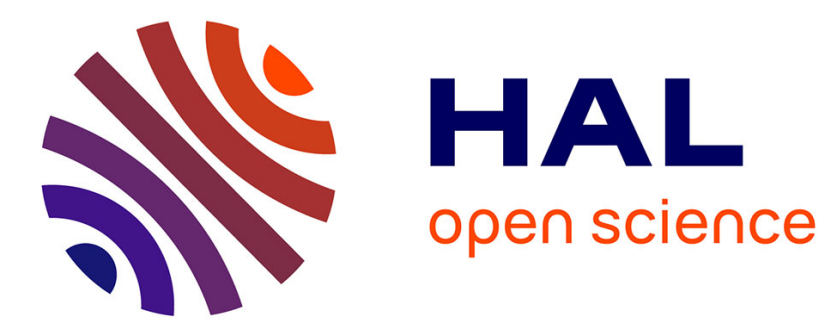

\title{
Hypocoercivity without confinement
}

Emeric Bouin, Jean Dolbeault, Stéphane Mischler, Clément Mouhot, Christian Schmeiser

\section{To cite this version:}

Emeric Bouin, Jean Dolbeault, Stéphane Mischler, Clément Mouhot, Christian Schmeiser. Hypocoercivity without confinement. 2017. hal-01575501v2

\section{HAL Id: hal-01575501 \\ https://hal.science/hal-01575501v2}

Preprint submitted on 5 Oct 2017 (v2), last revised 20 Sep 2019 (v4)

HAL is a multi-disciplinary open access archive for the deposit and dissemination of scientific research documents, whether they are published or not. The documents may come from teaching and research institutions in France or abroad, or from public or private research centers.
L'archive ouverte pluridisciplinaire HAL, est destinée au dépôt et à la diffusion de documents scientifiques de niveau recherche, publiés ou non, émanant des établissements d'enseignement et de recherche français ou étrangers, des laboratoires publics ou privés. 


\title{
HYPOCOERCIVITY WITHOUT CONFINEMENT
}

\author{
by \\ Emeric Bouin, Jean Dolbeault, Stéphane Mischler, \\ Clément Mouhot \& Christian Schmeiser
}

\begin{abstract}
In this paper, hypocoercivity methods are applied to linear kinetic equations with mass conservation and without confinement, in order to prove that the long-time behavior has algebraic decay as in the case of the heat equation. Two alternative approaches are developed: an analysis based on decoupled Fourier modes and a direct approach where, instead of the Poincaré inequality for the Dirichlet form, Nash's inequality is employed. The first approach is also used to provide a proof of exponential decay to equilibrium on the flat torus. The results are obtained on a space with exponential weights and then extended to larger function spaces by a factorization method. The optimality of the rates is discussed. Algebraic rates of decay on the whole space are also improved when the initial datum has zero average.
\end{abstract}

\section{Introduction}

We consider the Cauchy problem

$$
\partial_{t} f+v \cdot \nabla_{x} f=\mathrm{L} f, \quad f(0, x, v)=f_{0}(x, v)
$$

for a distribution function $f(t, x, v)$, with position variable in the whole space, $x \in \mathbb{R}^{d}$, or in the flat $d$-dimensional torus, $x \in \mathbb{T}^{d}$ (represented by the box $[0,2 \pi)^{d}$ with periodic boundary conditions), with velocity variable $v \in \mathbb{R}^{d}$, and with time $t \geq 0$. Concerning the collision operator $\mathrm{L}$, we shall consider two main cases:

(a) Fokker-Planck collision operator:

$$
\mathrm{L} f=\nabla_{\nu} \cdot\left[M \nabla_{\nu}\left(M^{-1} f\right)\right],
$$

(b) Scattering collision operator:

$$
\mathrm{L} f=\int_{\mathbb{R}^{d}} \sigma\left(\cdot, v^{\prime}\right)\left(f\left(v^{\prime}\right) M(\cdot)-f(\cdot) M\left(v^{\prime}\right)\right) d v^{\prime} .
$$

2010 Mathematics Subject Classification. - Primary: 82C40. Secondary: 76P05; 35H10; 35K65; 35P15; 35Q84.

Key words and phrases. - Hypocoercivity; linear kinetic equations; Fokker-Planck operator; scattering operator; transport operator; Fourier mode decomposition; Nash's inequality; factorization method; Green's function; micro/macro decomposition; diffusion limit.

Corresponding author: Émeric Bouin. 
A typical example of a local equilibrium $M$ is the Gaussian function

$$
M(\nu)=\frac{e^{-\frac{1}{2}|\nu|^{2}}}{(2 \pi)^{d / 2}}
$$

but our results apply to more general functions $M$ under assumptions that will be listed below. Without any further notice, we shall assume that

$$
\int_{\mathbb{R}^{d}} M(v) d v=1
$$

and it will be convenient to consider the measure

$$
d \gamma=\gamma(\nu) d v:=\frac{d v}{M(v)} .
$$

For simplicity, we shall say that $\gamma$ is an exponential weight even when $M$ is not a Gaussian function, if

$$
\lim _{|\nu| \rightarrow \infty} \frac{|\nu|^{k}}{\gamma(\nu)}=\lim _{|\nu| \rightarrow \infty} M(\nu)|\nu|^{k}=0 \quad \forall k \in(d, \infty) .
$$

In Case (b), the scattering rate $\sigma$ is assumed to satisfy

$$
1 \leq \sigma\left(\nu, v^{\prime}\right) \leq \bar{\sigma} \quad \forall v, v^{\prime} \in \mathbb{R}^{d}
$$

for some positive, finite $\bar{\sigma}$. Note that the lower bound on $\sigma$ can be achieved without loss of generality by rescalings, as soon as it is uniformly bounded away from 0. With $\sigma \equiv 1$, Case (b) includes the relaxation - or linear BGK - operator $\mathrm{L} f=M \rho_{f}-f$, with position density defined by

$$
\rho_{f}(t, x)=\int_{\mathbb{R}^{d}} f(t, x, v) d v .
$$

Note that micro-reversibility, i.e., the symmetry of $\sigma$, is not required. A computation shows that the general scattering operator can be compared to the linear BGK operator because

$$
\int_{\mathbb{R}^{d}}|\mathrm{~L} f|^{2} d \gamma \leq 4 \bar{\sigma}^{2} \int_{\mathbb{R}^{d}}\left|M \rho_{f}-f\right|^{2} d \gamma .
$$

Both cases, (a) and (b), share the properties that the null space of $L$ is spanned by the local equilibrium $M$, and that $\mathrm{L}$ only acts on the velocity variable. Under the requirement

$$
\int_{\mathbb{R}^{d}}\left(\sigma\left(v, v^{\prime}\right)-\sigma\left(v^{\prime}, v\right)\right) M\left(v^{\prime}\right) d v^{\prime}=0 \quad \forall v \in \mathbb{R}^{d}
$$

in Case (b), both cases satisfy the local mass conservation, i.e., $\int_{\mathbb{R}^{d}} \mathrm{~L} f d v=0$.

Throughout this paper, we shall assume that $M \in \mathrm{L}^{1}\left(\mathbb{R}^{d}\right)$ is nonnegative, continuous, radially symmetric (in the sense that $M(v)$ only depends on $|v|$ ) and that the second order moments of $M$ are bounded. A local equilibrium with these properties will be called an admissible local equilibrium. Let us define

$$
\Theta=\frac{1}{d} \int_{\mathbb{R}^{d}}|v|^{2} M(v) d v=\int_{\mathbb{R}^{d}}(\nu \cdot \mathrm{e})^{2} M(v) d v
$$


for an arbitrary e $\in \mathbb{S}^{d-1}$. Note that $M$ being radially symmetric, $\Theta$ does not depend on e. By the radial symmetry assumption again, we know that

$$
\int_{\mathbb{R}^{d}} v \otimes v M(v) d v=\Theta \mathrm{Id}
$$

If $M$ is the normalized Gaussian function, we find that $\Theta=1$. In Case (a), we further assume that

$$
\theta=\frac{1}{d}\left\|\nabla_{v} M\right\|_{\mathrm{L}^{2}(d \gamma)}^{2}=\frac{4}{d} \int_{\mathbb{R}^{d}}\left|\nabla_{v} \sqrt{M}\right|^{2} d v<\infty
$$

and adopt the convention that $\bar{\sigma}=\frac{1}{2} \sqrt{\theta / \Theta}$. When $M$ is the normalized Gaussian function, we find that $\theta=1$.

We assume that the operator $L$ satisfies the microscopic coercivity property

$$
-\int_{\mathbb{R}^{d}} \mathrm{~L} F(v) F(v) d \gamma \geq \lambda_{m} \int_{\mathbb{R}^{d}}\left(F-M \rho_{F}\right)^{2} d \gamma
$$

for some $\lambda_{m}>0$. In Case (a), Assumption (P) is equivalent to the Poincaré inequality

$$
\int_{\mathbb{R}^{d}}|\nabla u|^{2} M d v \geq \lambda_{m} \int_{\mathbb{R}^{d}}\left(u-\int_{\mathbb{R}^{d}} u M d v\right)^{2} M d v
$$

for all $u=M^{-1} F \in \mathrm{H}^{1}(M d v)$. The inequality holds with optimal constant $\lambda_{m}=1$ if $M$ is the normalized Gaussian function. We refer to $[29,2]$ for further details. In Case (b), Assumption (P) means

$$
\frac{1}{2} \iint_{\mathbb{R}^{d} \times \mathbb{R}^{d}} \sigma\left(v, v^{\prime}\right) M(v) M\left(v^{\prime}\right)\left(u(v)-u\left(v^{\prime}\right)\right)^{2} d v^{\prime} d v \geq \lambda_{m} \int_{\mathbb{R}^{d}}\left(u-\rho_{u M}\right)^{2} M d v
$$

for all $u=M^{-1} F \in \mathrm{L}^{2}(M d v)$. By [8, Proposition 2.2], the inequality arises as a consequence of (2). In the special case $\sigma \equiv 1$, notice that $\lambda_{m}=1$.

Summarizing our hypotheses on the collision operator, we shall say that $L$ satisfies Assumption $(\mathrm{H})$ if it is given either by Case (a) or by Case (b) and:

$\triangleright$ Case (a): (6) and (7) hold with $\theta<\infty$ and $\lambda_{m}>0$ (by convention: $\bar{\sigma}=\frac{1}{2} \sqrt{\theta / \Theta}$ ), $\triangleright$ Case (b): (2) holds for some $\bar{\sigma}>0$.

In order to perform a mode-by-mode hypocoercivity analysis, we introduce the Fourier representation with respect to $x$,

$$
f(t, x, v)=\int_{\mathbb{R}^{d}} \hat{f}(t, \xi, v) e^{-i x \cdot \xi} d \mu(\xi),
$$

where $d \mu(\xi)=(2 \pi)^{-d} d \xi$ and $d \xi$ is the Lesbesgue measure for the case $x \in \mathbb{R}^{d}$, whereas $d \mu(\xi)=(2 \pi)^{-d} \sum_{z \in \mathbb{Z}^{d}} \delta(\xi-z)$ is discrete for $x \in \mathbb{T}^{d}$. The normalization of $d \mu(\xi)$ is chosen such that Parseval's identity in the discrete case and Plancherel's formula when $x \in \mathbb{R}^{d}$ read

$$
\|f(t, \cdot, v)\|_{\mathrm{L}^{2}(d x)}=\|\hat{f}(t, \cdot, v)\|_{\mathrm{L}^{2}(d \mu(\xi))} .
$$

The Cauchy problem (1) in Fourier variables is now decoupled in the $\xi$-direction:

$$
\partial_{t} \hat{f}+\mathrm{T} \hat{f}=\mathrm{L} \hat{f}, \quad \hat{f}(0, \xi, v)=\hat{f}_{0}(\xi, v)
$$


where

$$
\mathrm{T} \hat{f}=i(\nu \cdot \xi) \hat{f}
$$

The outline of this paper goes as follows. In Section 2, we slightly strengthen the abstract hypocoercivity result of [10] by allowing complex Hilbert spaces and by providing explicit formulas for the coefficients in the decay rate. This result (Theorem 3) is applied for a given $\xi$ in Theorem 4 to the Fourier transformed problem (8), where integrals are computed with respect to the measure $d \gamma$ in the velocity variable $v$ : the mode-by-mode hypocoercivity estimate produces an exponential convergence rate in the case of the flat torus $\mathbb{T}^{d}$ (see Theorem 7) or explicit algebraic decay rates in the whole space case (see Theorem 1).

In Section 3, we state a special case of the factorization result of [16] (see Theorem 5). The estimate of Theorem 4, corresponding to the exponential weight $\gamma$, can be extended to larger spaces in Theorem 6 . This extension result corresponds to weights with a slower growth as $|\nu| \rightarrow \infty$ and goes as follows. Let

$$
d \gamma_{k}:=\gamma_{k}(v) d v \text { where } \gamma_{k}(v)=\left(1+|v|^{2}\right)^{k / 2} .
$$

We will systematically assume that $k>d$ in order to ensure that $\gamma_{k}^{-1} \in \mathrm{L}^{1}\left(\mathbb{R}^{d}\right)$. We adopt the convention that $\gamma_{\infty}=\gamma=M^{-1}$, so that the condition $k \in(d, \infty]$ covers the case of weights with a growth of the order of $|\nu|^{k}$ when $k$ is finite and weights with, typically, an $O\left(e^{|v|^{2} / 2}\right)$ growth when $k=\infty$. Hypocoercivity results based on the mode-by-mode analysis with measure $d \gamma_{k}$ are proved in Section 4 in the case of the flat torus $\mathbb{T}^{d}$ (Theorem 7) and of the whole space (first proof of Theorem 1). Our first main result of decay in the whole space case is summarized as follows.

Theorem 1. - Let us consider an admissible $M$ and a collision operator $\mathrm{L}$ satisfying Assumption $(\mathrm{H})$. Assume that $x \in \mathbb{R}^{d}, k \in(d, \infty]$ and $\gamma$ has an exponential growth if $k<\infty$. There exists a constant $C>0$ such that the solution $f$ of (1) with initial datum $f_{0} \in \mathrm{L}^{2}\left(d \gamma_{k} ; \mathrm{L}^{1}(d x)\right) \cap \mathrm{L}^{2}\left(d x d \gamma_{k}\right)$ satisfies

$$
\|f(t, \cdot, \cdot)\|_{\mathrm{L}^{2}\left(d x d \gamma_{k}\right)}^{2} \leq C\left(\left\|f_{0}\right\|_{\mathrm{L}^{2}\left(d x d \gamma_{k}\right)}^{2}+\left\|f_{0}\right\|_{\mathrm{L}^{2}\left(d \gamma_{k} ; \mathrm{L}^{1}(d x)\right)}^{2}\right)(1+t)^{-\frac{d}{2}} \quad \forall t \geq 0 .
$$

As we shall see in the Appendix A by computing Green's function in a simple case, the rates of Theorem 1 are optimal at least in the case of the kinetic FokkerPlanck collision operator associated with the normalized Gaussian function: the decay rate is the rate of the heat equation on $\mathbb{R}^{d}$. Our method is consistent with the diffusion limit and provides estimates which are asymptotically uniform: see Appendix B.

In Section 5, an hypocoercivity method in which Poincaré's inequality, or the so-called macroscopic coercivity condition, is replaced by Nash's inequality provides an alternative proof of Theorem 1. Notice that the direct approach by Nash's inequality is also applicable to problems with non-constant coefficients like scattering operators with $x$-dependent scattering rates $\sigma$, or Fokker-Planck operators with $x$-dependent diffusion constants like $\nabla_{\nu} \cdot\left(\mathscr{D}(x) M \nabla_{\nu}\left(M^{-1} f\right)\right)$. 
Inspired by the heat equation, the result of Theorem 1 suggests to investigate the case of a sign changing distribution function with zero average using Fourier techniques. Our second main result of decay in the whole space goes as follows.

Theorem 2. - Let us consider an admissible $M$ and a collision operator $\mathrm{L}$ satisfying Assumption (H). Let $x \in \mathbb{R}^{d}, k \in(d, \infty)$ and assume that $\gamma$ has an exponential growth. Then there exists a constant $c_{k}>0$ such that, for any $f_{0} \in \mathrm{L}_{\mathrm{loc}}^{1}\left(\mathbb{R}^{d} \times \mathbb{R}^{d}\right)$ with

$$
\iint_{\mathbb{R}^{d} \times \mathbb{R}^{d}} f_{0}(x, v) d x d v=0
$$

and $\mathscr{C}_{0}:=\left\|f_{0}\right\|_{\mathrm{L}^{2}\left(d \gamma_{k+2} ; \mathrm{L}^{1}(d x)\right)}^{2}+\left\|f_{0}\right\|_{\mathrm{L}^{2}\left(d \gamma_{k} ; \mathrm{L}^{1}(|x| d x)\right)}^{2}+\left\|f_{0}\right\|_{\mathrm{L}^{2}\left(d x d \gamma_{k}\right)}^{2}<\infty$, the solution $f$ of (1) with initial datum $f_{0}$ satisfies

$$
\|f(t, \cdot, \cdot)\|_{\mathrm{L}^{2}\left(d x d \gamma_{k}\right)}^{2} \leq c_{k} \mathscr{C}_{0}(1+t)^{-\left(1+\frac{d}{2}\right)} \quad \forall t \geq 0 .
$$

We conclude this introduction by a brief review of the literature. On the whole Euclidean space, large time asymptotics are governed by the heat equation: we refer to [31] for recent lecture notes on available techniques for capturing the large time asymptotics of the heat equation. Some of our results make a clear link with the heat flow seen as the diffusion limit of the kinetic equation. We refer to [21] for recent results on the diffusion, or overdamped, limit (also see Appendix B).

The mode-by-mode analysis is an extension of the hypocoercivity theory of [10], which has been inspired by [18], but is also close to the Kawashima compensating function method: see $[24, \mathbf{1 5}]$. We also refer to [12] where the Kawashima approach is applied to a particular case of the scattering model (b).

The word hypocoercivity was coined by T. Gallay and widely disseminated in

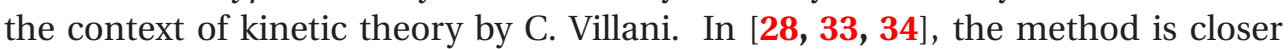
to the hypoellipticity theory, which simultaneously deals with regularity and large time properties of the solutions by considering a $\mathrm{H}^{1}$-norm (in $x$ and $v$ variables) and taking into account cross-terms. This is very well explained in [33, Section 3], but was already present in earlier works like [19]. The hypoellipticity theory has a long history in the context of the kinetic Fokker-Planck equation. One can refer for instance to $[\mathbf{1 3}, 19]$ and much earlier to Hörmander's theory [20]. The seed for such an approach can even be traced back to Kolmogorov's computation of Green's kernel for the kinetic Fokker-Planck equation in [25], which has been reconsidered in [22] and successfully applied, for instance, to the study of the Vlasov-PoissonFokker-Planck system in $[\mathbf{3 2}, \mathbf{5}]$.

Linear Boltzmann equations and BGK (Bhatnagar-Gross-Krook, see [4]) models also have a long history: we shall refer to [8] (also see [7]) for key mathematical properties, and to $[\mathbf{2 8}, \mathbf{1 8}]$ for first hypocoercivity results. In this paper we will mostly rely on $[\mathbf{9}, \mathbf{1 0}]$. However, among more recent contributions, one has to quote $[17,1,6]$ and also an approach based on the Fisher information which has recently been implemented in $[14,27]$.

With the exponential weight $\gamma=M^{-1}$, Theorem 7 can be obtained directly by the method of [10]. The mode-by-mode hypocoercivity point of view developed in Section 2 and the extension of Section 3 based on the method of [16] provides us with the result in an extended space with weights having only a polynomial 
growth in the velocity variable. For completeness, let us mention that recently the exponential growth issue was overcome for the Fokker-Planck case in $[23,26]$ by a different method. The improved decay rate for zero average solutions established in Theorem 2 generalizes to kinetic models similar results known for the heat equation, see for instance [26, Remark 3.2 (7)] or [3].

\section{Mode-by-mode hypocoercivity}

Let us consider the evolution equation

$$
\frac{d F}{d t}+\mathrm{T} F=\mathrm{L} F
$$

where $T$ and $L$ are respectively a general transport operator and a general linear collision operator. We shall use the abstract approach of [10]. Although the extension of the method to Hilbert spaces over complex numbers is rather straightforward, we carry it out here for completeness and also derive new explicit estimates on the decay rate.

Theorem 3. - Let $\mathrm{L}$ and $\mathrm{T}$ be closed linear operators in the complex Hilbert space $(\mathscr{H},\langle\cdot, \cdot\rangle)$ with induced norm $\|\cdot\|$. Assume that $\mathrm{L}$ is Hermitian and $\mathrm{T}$ is antiHermitian. Let $\Pi$ be the orthogonal projection onto the null space of $L$ and define

$$
\mathrm{A}:=\left(1+(\mathrm{T} \Pi)^{*} \mathrm{~T} \Pi\right)^{-1}(\mathrm{~T} \Pi)^{*}
$$

where* denotes the adjoint with respect to $\langle\cdot, \cdot\rangle$. We assume that positive constants $\lambda_{m}, \lambda_{M}$, and $C_{M}$ exist, such that, for any $F \in \mathscr{H}$, the following properties hold: $\triangleright$ microscopic coercivity:

$$
-\langle\mathrm{L} F, F\rangle \geq \lambda_{m}\|(1-\Pi) F\|^{2},
$$

$\triangleright$ macroscopic coercivity:

$$
\|\mathrm{\top} \Pi F\|^{2} \geq \lambda_{M}\|\Pi F\|^{2},
$$

$\triangleright$ parabolic macroscopic dynamics:

$$
\text { ПТП } F=0 \text {, }
$$

$\triangleright$ bounded auxiliary operators:

$$
\|\mathrm{AT}(1-\Pi) F\|+\|\mathrm{AL} F\| \leq C_{M}\|(1-\Pi) F\| .
$$

Then for any $t \geq 0$, we have

$$
\left\|e^{(\mathrm{L}-\mathrm{T}) t}\right\|^{2} \leq 3 e^{-\lambda t} \quad \text { where } \lambda=\frac{\lambda_{M}}{3\left(1+\lambda_{M}\right)} \min \left\{1, \lambda_{m}, \frac{\lambda_{m} \lambda_{M}}{\left(1+\lambda_{M}\right) C_{M}^{2}}\right\} .
$$

In the applications, the macroscopic coercivity, in practice a Poincaré inequality, holds only for functions with zero average.

Proof. - For some $\delta>0$ to be determined later, the Lyapunov functional

$$
\mathrm{H}[F]:=\frac{1}{2}\|F\|^{2}+\delta \operatorname{Re}\langle\mathrm{A} F, F\rangle
$$


is such that $\frac{d}{d t} \mathrm{H}[F]=-\mathrm{D}[F]$ if $F$ solves (11), with

$\mathrm{D}[F]:=-\langle\mathrm{L} F, F\rangle+\delta\langle\mathrm{AT} \Pi F, F\rangle+\delta \operatorname{Re}\langle\mathrm{AT}(1-\Pi) F, F\rangle-\delta \operatorname{Re}\langle\mathrm{TA} F, F\rangle-\delta \operatorname{Re}\langle\mathrm{AL} F, F\rangle$.

Since the Hermitian operator АТП can be interpreted as the application of the map $z \mapsto(1+z)^{-1} z$ to $(Т \Pi)^{*}$ ТП, as a consequence of the spectral theorem [30, Theorem VII.2, p. 225], the conditions (H1) and (H2) imply that

$$
-\langle\mathrm{L} F, F\rangle+\delta\langle\mathrm{AT} \Pi F, F\rangle \geq \lambda_{m}\|(1-\Pi) F\|^{2}+\frac{\delta \lambda_{M}}{1+\lambda_{M}}\|\Pi F\|^{2} .
$$

As in [10, Lemma 1], if $G=\mathrm{A} F$, i.e., $G+(\mathrm{T} \Pi)^{*} \mathrm{~T} \Pi G=(\mathrm{T} \Pi)^{*} F$, a Cauchy-Schwarz estimate applied to

$$
\|\mathrm{A} F\|^{2}+\|\mathrm{TA} F\|^{2}=\left\langle G, G+(\mathrm{T} \Pi)^{*} \mathrm{~T} \Pi G\right\rangle=\left\langle G,(\mathrm{~T} \Pi)^{*} F\right\rangle=\langle\mathrm{TA} F,(1-\Pi) F\rangle
$$

shows that

$$
\|\mathrm{TA} F\|^{2} \leq 2\|\mathrm{~A} F\|^{2}+\|\mathrm{TA} F\|^{2}=2\|G\|^{2}+\|\mathrm{T} G\|^{2} \leq\|(1-\Pi) F\|^{2}
$$

and establishes for any $\delta<1$ the norm equivalence of $\mathrm{H}[F]$ and $\|F\|^{2}$, that is,

$$
\frac{1}{2}(1-\delta)\|F\|^{2} \leq \mathrm{H}[F] \leq \frac{1}{2}(1+\delta)\|F\|^{2} .
$$

With $X:=\|(1-\Pi) F\|$ and $Y:=\|\Pi F\|$, it follows from (H4) that

$$
\mathrm{D}[F] \geq\left(\lambda_{m}-\delta\right) X^{2}+\frac{\delta \lambda_{M}}{1+\lambda_{M}} Y^{2}-\delta C_{M} X Y .
$$

The choice $\delta=\frac{1}{2} \min \left\{1, \lambda_{m}, \frac{\lambda_{m} \lambda_{M}}{\left(1+\lambda_{M}\right) C_{M}^{2}}\right\}$ implies that

$$
\mathrm{D}[F] \geq \frac{\lambda_{m}}{4} X^{2}+\frac{\delta \lambda_{M}}{2\left(1+\lambda_{M}\right)} Y^{2} \geq \frac{1}{4} \min \left\{\lambda_{m}, \frac{2 \delta \lambda_{M}}{1+\lambda_{M}}\right\}\|F\|^{2} \geq \frac{2 \delta \lambda_{M}}{3\left(1+\lambda_{M}\right)} \mathrm{H}[F] .
$$

With $\lambda$ defined in (12), using $\delta \leq 1 / 2$ and $(1+\delta) /(1-\delta) \leq 3$, we get

$$
\|F(t)\|^{2} \leq \frac{2}{1-\delta} \mathrm{H}[F](t) \leq \frac{1+\delta}{1-\delta} e^{-\lambda t}\|F(0)\|^{2} \leq 3 e^{-\lambda t}\|F(0)\|^{2} .
$$

For any fixed $\xi \in \mathbb{R}^{d}$, let us apply Theorem 3 to (8) with

$$
\mathscr{H}=\mathrm{L}^{2}(d \gamma), \quad\|F\|^{2}=\int_{\mathbb{R}^{d}}|F|^{2} d \gamma, \quad \Pi F=M \int_{\mathbb{R}^{d}} F d v=M \rho_{F}
$$

and $\mathrm{T}$ given by (9). We recall that $\Theta$ and $\theta$ are defined by (4) and (6).

Theorem 4. - Let us consider an admissible $M$ and a collision operator $L$ satisfying Assumption $(\mathrm{H})$, and take $\xi \in \mathbb{R}^{d}$. If $\hat{f}$ is a solution of (8) such that $\hat{f}_{0}(\xi, \cdot) \in$ $\mathrm{L}^{2}(d \gamma)$, then for any $t \geq 0$, we have

where

$$
\|\hat{f}(t, \xi, \cdot)\|_{\mathrm{L}^{2}(d \gamma)}^{2} \leq 3 e^{-\mu_{\xi} t}\left\|\hat{f}_{0}(\xi, \cdot)\right\|_{\mathrm{L}^{2}(d \gamma)}^{2}
$$

$$
\mu_{\xi}:=\frac{\Lambda|\xi|^{2}}{1+|\xi|^{2}} \quad \text { and } \quad \Lambda=\frac{\Theta}{3 \max \{1, \Theta\}} \min \left\{1, \frac{\lambda_{m} \Theta}{\kappa^{2}+\Theta}\right\}
$$

with $\kappa=2 \bar{\sigma} \sqrt{\Theta}$ (i.e., $\kappa=\sqrt{\theta}$ in Case (a)). 
Proof. - We check that the assumptions of Theorem 3 are satisfied with $F=\hat{f}$. As shown in the introduction, Assumption (P) holds and corresponds to (H1). Concerning the macroscopic coercivity (H2), since

$$
\mathrm{T} \Pi F=i(\nu \cdot \xi) \rho_{F} M,
$$

one has

$$
\|\mathrm{T} \Pi F\|^{2}=\left|\rho_{F}\right|^{2} \int_{\mathbb{R}^{d}}|\nu \cdot \xi|^{2} M(v) d v=\Theta|\xi|^{2}\left|\rho_{F}\right|^{2}=\Theta|\xi|^{2}\|\Pi F\|^{2},
$$

and thus (H2) holds with $\lambda_{M}=\Theta|\xi|^{2}$. By assumption $M(\nu)$ depends only on $|\nu|$, so it is unbiased: $\int_{\mathbb{R}^{d}} v M(v) d v=0$, which means that (H3) holds. It remains to prove that (H4) also holds.

The operator $\mathrm{A}$ is given by

$$
\mathrm{A} F=\frac{-i \xi \cdot \int_{\mathbb{R}^{d}} v^{\prime} F\left(\nu^{\prime}\right) d v^{\prime}}{1+\Theta|\xi|^{2}} M
$$

and satisfies the estimate

$$
\begin{aligned}
&\|\mathrm{A} F\|=\|\mathrm{A}(1-\Pi) F\| \leq \frac{1}{1+\Theta|\xi|^{2}} \int_{\mathbb{R}^{d}} \frac{|(1-\Pi) F|}{\sqrt{M}}|v \cdot \xi| \sqrt{M} d v \\
& \leq \frac{1}{1+\Theta|\xi|^{2}}\|(1-\Pi) F\|\left(\int_{\mathbb{R}^{d}}(\nu \cdot \xi)^{2} M d v\right)^{1 / 2} \\
&=\frac{\sqrt{\Theta}|\xi|}{1+\Theta|\xi|^{2}}\|(1-\Pi) F\| .
\end{aligned}
$$

In Case (b) the collision operator $L$ is bounded according to (3):

$$
\|L F\|^{2} \leq 4 \bar{\sigma}^{2}\|(1-\Pi) F\|^{2} .
$$

For estimating $A L$ in Case (a), we note that

$$
\int_{\mathbb{R}^{d}} v \mathrm{LF} d v=2 \int_{\mathbb{R}^{d}} \nabla_{v} \sqrt{M} \frac{F}{\sqrt{M}} d v
$$

and obtain as above that

$$
\|\mathrm{AL} F\| \leq \frac{2}{1+\Theta|\xi|^{2}} \int_{\mathbb{R}^{d}} \frac{|(1-\Pi) F|}{\sqrt{M}}\left|\xi \cdot \nabla_{\nu} \sqrt{M}\right| d \nu \leq \frac{\sqrt{\theta}|\xi|}{1+\Theta|\xi|^{2}}\|(1-\Pi) F\| .
$$

For both cases we finally obtain

$$
\|\mathrm{ALF}\| \leq \frac{\kappa|\xi|}{1+\Theta|\xi|^{2}}\|(1-\Pi) F\|
$$

where $\kappa=\sqrt{\theta}$ in Case (a) and $\kappa=2 \bar{\sigma} \sqrt{\Theta}$ in Case (b). Similarly we can estimate

$$
\mathrm{TA} F(v)=-\frac{(\nu \cdot \xi) M}{1+\Theta|\xi|^{2}} \int_{\mathbb{R}^{d}}\left(v^{\prime} \cdot \xi\right)(1-\Pi) F\left(v^{\prime}\right) d v^{\prime}
$$

by

$$
\|\mathrm{TA} F\| \leq \frac{\Theta|\xi|^{2}}{1+\Theta|\xi|^{2}}\|(1-\Pi) F\|,
$$

meaning that we have proven (H4) with $C_{M}=\frac{\kappa|\xi|+\Theta|\xi|^{2}}{1+\Theta|\xi|^{2}}$. 
With the elementary estimates

$$
\frac{\Theta|\xi|^{2}}{1+\Theta|\xi|^{2}} \geq \frac{\Theta}{\max \{1, \Theta\}} \frac{|\xi|^{2}}{1+|\xi|^{2}} \quad \text { and } \quad \frac{\lambda_{M}}{\left(1+\lambda_{M}\right) C_{M}^{2}}=\frac{\Theta\left(1+\Theta|\xi|^{2}\right)}{(\kappa+\Theta|\xi|)^{2}} \geq \frac{\Theta}{\kappa^{2}+\Theta} \text {, }
$$

the proof is completed using (12).

\section{Enlarging the space by factorization}

Square integrability against the inverse of the local equilibrium $M$ is a rather restrictive assumption on the initial datum. In this section it will be relaxed with the help of the abstract factorization method of [16] in a simple case (factorization of order 1) that we state and prove here in a special case, for the convenience of the reader.

Theorem 5. - Let $\mathscr{B}_{1}, \mathscr{B}_{2}$ be Banach spaces and let $\mathscr{B}_{2}$ be continuously imbedded in $\mathscr{B}_{1}$, i.e., $\|\cdot\|_{1} \leq c_{1}\|\cdot\|_{2}$. Let $\mathfrak{B}$ and $\mathfrak{A}+\mathfrak{B}$ be the generators of the strongly continuous semigroups $e^{\mathfrak{B} t}$ and $e^{(\mathfrak{A}+\mathfrak{B}) t}$ on $\mathscr{B}_{1}$. Assume that there are positive constants $c_{2}, c_{3}, c_{4}, \lambda_{1}$ and $\lambda_{2}$ such that, for all $t \geq 0$,

$$
\left\|e^{(\mathfrak{A}+\mathfrak{B}) t}\right\|_{2 \rightarrow 2} \leq c_{2} e^{-\lambda_{2} t}, \quad\left\|e^{\mathfrak{B} t}\right\|_{1 \rightarrow 1} \leq c_{3} e^{-\lambda_{1} t}, \quad\|\mathfrak{A}\|_{1 \rightarrow 2} \leq c_{4},
$$

where $\|\cdot\|_{i \rightarrow j}$ denotes the operator norm for linear mappings from $\mathscr{B}_{i}$ to $\mathscr{B}_{j}$. Then there exists a positive constant $C=C\left(c_{1}, c_{2}, c_{3}, c_{4}\right)$ such that, for all $t \geq 0$,

$$
\left\|e^{(\mathfrak{A}+\mathfrak{B}) t}\right\|_{1 \rightarrow 1} \leq \begin{cases}C\left(1+\left|\lambda_{1}-\lambda_{2}\right|^{-1}\right) e^{-\min \left\{\lambda_{1}, \lambda_{2}\right\} t} & \text { for } \lambda_{1} \neq \lambda_{2}, \\ C(1+t) e^{-\lambda_{1} t} & \text { for } \lambda_{1}=\lambda_{2} .\end{cases}
$$

Proof. - Integrating the identity $\frac{d}{d s}\left(e^{(\mathfrak{A}+\mathfrak{B}) s} e^{\mathfrak{B}(t-s)}\right)=e^{(\mathfrak{A}+\mathfrak{B}) s} \mathfrak{A} e^{\mathfrak{B}(t-s)}$ with respect to $s \in[0, t]$ gives

$$
e^{(\mathfrak{A}+\mathfrak{B}) t}=e^{\mathfrak{B} t}+\int_{0}^{t} e^{(\mathfrak{A}+\mathfrak{B}) s} \mathfrak{A} e^{\mathfrak{B}(t-s)} d s .
$$

The proof is completed by the straightforward computation

$$
\begin{aligned}
\left\|e^{(\mathfrak{A}+\mathfrak{B}) t}\right\|_{1 \rightarrow 1} \leq c_{3} e^{-\lambda_{1} t}+c_{1} \int_{0}^{t}\left\|e^{(\mathfrak{A}+\mathfrak{B}) s} \mathfrak{A} e^{\mathfrak{B}(t-s)}\right\|_{1 \rightarrow 2} d s & \\
& \leq c_{3} e^{-\lambda_{1} t}+c_{1} c_{2} c_{3} c_{4} e^{-\lambda_{1} t} \int_{0}^{t} e^{\left(\lambda_{1}-\lambda_{2}\right) s} d s .
\end{aligned}
$$

Theorem 5 applies to (8) with the spaces $\mathscr{B}_{1}=\mathrm{L}^{2}\left(d \gamma_{k}\right)$ and $\mathscr{B}_{2}=\mathrm{L}^{2}(d \gamma)$ corresponding to the weights defined by (10). The exponential growth assumption on $\gamma$ guarantees that $\mathscr{B}_{2}$ is continuously imbedded in $\mathscr{B}_{1}$.

Theorem 6. - Let us consider an admissible $M$ and a collision operator $\mathrm{L}$ satisfying Assumption $(\mathrm{H})$. We assume that $k \in(d, \infty], \gamma$ has an exponential growth if $k<\infty$, take $\xi \in \mathbb{R}^{d}$ and consider the solution $\hat{f}$ of (8) with initial datum $\hat{f}_{0}(\xi, \cdot) \in$ $\mathrm{L}^{2}\left(d \gamma_{k}\right)$. Then, there exists a constant $C=C(k, d, \bar{\sigma})$ such that, with $\mu_{\xi}$ given by (15),

$$
\|\hat{f}(t, \xi, \cdot)\|_{\mathrm{L}^{2}\left(d \gamma_{k}\right)}^{2} \leq C e^{-\mu_{\xi} t}\left\|\hat{f}_{0}(\xi, \cdot)\right\|_{\mathrm{L}^{2}\left(d \gamma_{k}\right)}^{2} \quad \forall t \geq 0 .
$$


Proof. - In Case (a), let us define $\mathfrak{A}$ and $\mathfrak{B}$ by $\mathfrak{A} F=N \chi_{R} F$ and $\mathfrak{B} F=-i(\nu \cdot \xi) F+$ $\mathrm{L} F-\mathfrak{A} F$, where $N$ and $R$ are two positive constants, $\chi$ is a smooth function such that $\mathbb{1}_{B_{1}} \leq \chi \leq \mathbb{1}_{B_{2}}$, where $B_{r}$ is the centred ball of radius $r$, and $\chi_{R}:=\chi(\cdot / R)$. For any $R$ and $N$ large enough, it has been established in [26, Lemma 3.8] that

$$
\int_{\mathbb{R}^{d}}(\mathrm{~L}-\mathfrak{A})(F) F d \gamma_{k} \leq-\lambda_{1} \int_{\mathbb{R}^{d}} F^{2} d \gamma_{k}
$$

for some $\lambda_{1}>0$ if $k>d$. The boundedness of $\mathfrak{A}: \mathscr{B}_{1} \rightarrow \mathscr{B}_{2}$ follows from the compactness of the support of $\chi$ and Theorem 5 applies with $\lambda_{2}=\mu_{\xi} / 2 \leq 1 / 4$, where $\mu_{\xi}$ is given by (15).

In Case (b), we consider $\mathfrak{A}$ and $\mathfrak{B}$ such that

$$
\begin{aligned}
& \mathfrak{A} F(v)=M(v) \int_{\mathbb{R}^{d}} \sigma\left(v, v^{\prime}\right) F\left(v^{\prime}\right) d v^{\prime}, \\
& \mathfrak{B} F(v)=-\left[i(\nu \cdot \xi)+\int_{\mathbb{R}^{d}} \sigma\left(\nu, v^{\prime}\right) M\left(v^{\prime}\right) d v^{\prime}\right] F(v) .
\end{aligned}
$$

The boundedness of $\mathfrak{A}: \mathscr{B}_{1} \rightarrow \mathscr{B}_{2}$ follows from (2) and

$$
\|\mathfrak{A} F\|_{\mathrm{L}^{2}(d \gamma)} \leq \bar{\sigma}\|F\|_{\mathrm{L}^{1}(d v)} \leq \bar{\sigma}\left(\int_{\mathbb{R}^{d}} \gamma_{k}^{-1} d v\right)^{1 / 2}\|F\|_{\mathrm{L}^{2}\left(d \gamma_{k}\right)} .
$$

Theorem 5 applies with $\lambda_{2}=\frac{\mu_{\xi}}{2} \leq \frac{1}{4}$ and $\lambda_{1}=1$ because $\int_{\mathbb{R}^{d}} \sigma\left(v, v^{\prime}\right) M\left(v^{\prime}\right) d v^{\prime} \geq 1$.

\section{Asymptotic behavior based on mode-by-mode hypocoercivity}

In this section we consider (1) in the direct variable $x$ of position and use the estimates of Theorem 4 (with the exponential weight $\gamma=M^{-1}$ ) and Theorem 6 (for weights with $O\left(|v|^{k}\right)$ growth) to get exponential convergence results in $\mathbb{T}^{d}$ (Section 4.1) and algebraic decay rates in $\mathbb{R}^{d}$ (Section 4.2).

4.1. Exponential convergence to equilibrium in $\mathbb{T}^{d}$. - The unique global equilibrium in the case $x \in \mathbb{T}^{d}$ is given by

$$
f_{\infty}(x, v)=\rho_{\infty} M(v) \quad \text { with } \quad \rho_{\infty}=\frac{1}{\left|\mathbb{T}^{d}\right|} \iint_{\mathbb{T}^{d} \times \mathbb{R}^{d}} f_{0} d x d v .
$$

Theorem 7. - Assume that $k \in(d, \infty]$ and $\gamma$ has an exponential growth if $k<\infty$. We consider an admissible $M$, a collision operator $\mathrm{L}$ satisfying Assumption $(\mathrm{H})$, and $\Lambda$ given by (15). There exists a positive constant $C_{k}$ such that the solution $f$ of (1) on $\mathbb{T}^{d} \times \mathbb{R}^{d}$ with initial datum $f_{0} \in \mathrm{L}^{2}\left(d x d \gamma_{k}\right)$ satisfies

$$
\left\|f(t, \cdot, \cdot)-f_{\infty}\right\|_{\mathrm{L}^{2}\left(d x d \gamma_{k}\right)} \leq C_{k}\left\|f_{0}-f_{\infty}\right\|_{\mathrm{L}^{2}\left(d x d \gamma_{k}\right)} e^{-\frac{\Lambda}{4} t} \quad \forall t \geq 0 .
$$

Proof. - If we represent the flat torus $\mathbb{T}^{d}$ by the box $[0,2 \pi)^{d}$ with periodic boundary conditions, the Fourier variable satisfies $\xi \in \mathbb{Z}^{d}$. For $\xi=0$, the microscopic coercivity (see Section 2) implies

$$
\left\|\hat{f}(t, 0, \cdot)-\hat{f}_{\infty}(0, \cdot)\right\|_{\mathrm{L}^{2}(d \gamma)} \leq\left\|\hat{f}_{0}(0, \cdot)-\hat{f}_{\infty}(0, \cdot)\right\|_{\mathrm{L}^{2}(d \gamma)} e^{-t} .
$$


For all other modes, $\hat{f}_{\infty}(\xi, \cdot)=0$ for any $\xi \neq 0$ (that is, for any $\xi$ such that $|\xi| \geq 1$ ). We can use Theorem 4 with $\mu_{\xi} \geq \Lambda / 2$, with the notations of (15). An application of Parseval's identity then proves the result with $\gamma=\gamma_{\infty}$, i.e. for $k=\infty$, and $C_{\infty}=\sqrt{3}$. This result can also be derived by directly applying Theorem 3 to (1), as in [10]. If $k$ is finite, the result with the weight $\gamma_{k}$ follows from Theorem 6 , for some $C_{k}>0$.

4.2. Algebraic decay rates in $\mathbb{R}^{d}$. - We give a first proof of Theorem 1 . We start with the result of Theorem 4 and Theorem 6 and integrate with respect to $\xi$. Let $C>0$ be a generic constant which is going to change from line to line. The Plancherel formula implies

$$
\|f(t, \cdot, \cdot)\|_{\mathrm{L}^{2}\left(d x d \gamma_{k}\right)}^{2} \leq C \int_{\mathbb{R}^{d}}\left(\int_{\mathbb{R}^{d}} e^{-\mu_{\xi} t}\left|\hat{f}_{0}\right|^{2} d \xi\right) d \gamma_{k} .
$$

We know that $\int_{|\xi| \leq 1} e^{-\mu_{\xi} t} d \xi \leq \int_{\mathbb{R}^{d}} e^{-\frac{\Lambda}{2}|\xi|^{2} t} d \xi=\left(\frac{2 \pi}{\Lambda t}\right)^{d / 2}$ and thus, for all $v \in \mathbb{R}^{d}$,

$$
\int_{|\xi| \leq 1} e^{-\mu_{\xi} t}\left|\hat{f}_{0}\right|^{2} d \xi \leq C\left\|f_{0}(\cdot, v)\right\|_{\mathrm{L}^{1}(d x)}^{2} \int_{\mathbb{R}^{d}} e^{-\frac{\Lambda}{2}|\xi|^{2} t} d \xi \leq C\left\|f_{0}(\cdot, v)\right\|_{\mathrm{L}^{1}(d x)}^{2} t^{-\frac{d}{2}}
$$

Using the fact that $\mu_{\xi} \geq \Lambda / 2$ when $|\xi| \geq 1$ and Plancherel's formula, we know that, for all $v \in \mathbb{R}^{d}$,

which completes the proof of Theorem 1 .

$$
\int_{|\xi|>1} e^{-\mu_{\xi} t}\left|\hat{f}_{0}\right|^{2} d \xi \leq C e^{-\frac{\Lambda}{2} t}\left\|f_{0}(\cdot, v)\right\|_{\mathrm{L}^{2}(d x)}^{2},
$$

\section{Algebraic decay rates in $\mathbb{R}^{d}$}

With a position variable $x \in \mathbb{R}^{d}$, we can rely on Nash's inequality [29],

$$
\|u\|_{\mathrm{L}^{2}(d x)}^{2} \leq \mathscr{C}_{\text {Nash }}\|u\|_{\mathrm{L}^{1}(d x)}^{\frac{4}{d+2}}\|\nabla u\|_{\mathrm{L}^{2}(d x)}^{\frac{2 d}{d+2}},
$$

to obtain optimal algebraic decay rates: see Section 5.1.

We can also state an improved Nash inequality

$$
\|u\|_{\mathrm{L}^{2}(d x)}^{2} \leq \mathscr{C}_{\star}\|x u\|_{\mathrm{L}^{1}(d x)}^{\frac{4}{d+4}}\|\nabla u\|_{\mathrm{L}^{2}(d x)}^{\frac{d+2}{d+4}}
$$

valid, with some positive constant $\mathscr{C}_{\star}$, for any $u \in \mathrm{H}^{1}(d x) \cap \mathrm{L}^{1}((1+|x|) d x)$ such that $\int_{\mathbb{R}^{d}} u d x=0$. The proof follows from a minor modification of Nash's original proof in [29] and uses Fourier variables. As a consequence, any solution of the heat equation with zero average decays in $\mathrm{L}^{2}(d x)$ like $O\left(t^{-\left(1+\frac{d}{2}\right)}\right)$ as $t \rightarrow+\infty$. In Section 5.2, we will give a proof of Theorem 2 for a solution of (1) with zero average, which directly relies on estimates in Fourier variables, in the spirit of Nash's proof.

5.1. Hypocoercivity and Nash's inequality. - Let us state a result similar to the one of Theorem 1, with slightly different norms. Here we shall abandon the Fourier variable $\xi$ and consider the direct variable $x \in \mathbb{R}^{d}$ : throughout this section, the transport operator on the position space is redefined as

$$
\mathrm{T} f=v \cdot \nabla_{x} f .
$$

We rely on the abstract setting of Section 2, applied to (1) with the scalar product $\langle\cdot, \cdot\rangle$ on $\mathrm{L}^{2}(d x d \gamma)$ and the induced norm $\|\cdot\|$. Notice that this norm includes the $x$ 
variable, which was not the case in the mode-by-mode analysis of Section 2 . It is then easy to check that $(\mathrm{T} \Pi) f=M \top \rho_{f}=v \cdot \nabla_{x} \rho_{f} M$, ( $\left.\mathrm{T} \Pi\right)^{*} f=-\nabla_{x} \cdot\left(\int_{\mathbb{R}^{d}} v f d v\right) M$ and $(\mathrm{T} \Pi)^{*}(\mathrm{~T} \Pi) f=-\Theta \Delta_{x} \rho_{f} M$ so that

$$
g=\mathrm{A} f=\left(1+(\mathrm{T} \Pi)^{*} \mathrm{~T} \Pi\right)^{-1}(\mathrm{~T} \Pi)^{*} f \quad \Longleftrightarrow \quad g=u M
$$

where $u-\Theta \Delta u=-\nabla_{x} \cdot\left(\int_{\mathbb{R}^{d}} v f d v\right)$. Since $M$ is unbiased, $\mathrm{A} f=\mathrm{A}(1-\Pi) f$. For some $\delta>0$ to be chosen later, we redefine the modified entropy by

$$
\mathrm{H}[f]:=\frac{1}{2}\|f\|^{2}+\delta\langle\mathrm{A} f, f\rangle .
$$

Theorem 8. - Let us consider an admissible $M$ such that, for an arbitrary e $\epsilon$ $\mathbb{S}^{d-1}, K^{2}=\int_{\mathbb{R}^{d}}(\nu \cdot \mathrm{e})^{4} M(v) d v<\infty$, and a collision operator $\mathrm{L}$ satisfying Assumption $(\mathrm{H})$ on $\mathbb{R}^{d}$. There exists a constant $C>0$ such that the solution $f$ of (1) with initial datum $f_{0} \in \mathrm{L}^{2}\left(d \gamma ; \mathrm{L}^{1}(d x)\right) \cap \mathrm{L}^{1}(d x d v)$ satisfies

$$
\mathrm{H}[f] \leq C\left(\mathrm{H}\left[f_{0}\right]+\left\|f_{0}\right\|_{\mathrm{L}^{1}(d x d v)}^{2}\right)(1+t)^{-\frac{d}{2}}, \quad \forall t \geq 0 .
$$

Note that $M$ being radially symmetric, $K$ does not depend on e. If $M$ is the normalized Gaussian function, we find that $K^{2}=3$.

Proof. - If $f$ solves (1), the time derivative of $\mathrm{H}[f(t, \cdot, \cdot)]$ is given by

(17) $\frac{d}{d t} \mathrm{H}[f]=\langle\mathrm{L} f, f\rangle-\delta\langle\mathrm{AT} \Pi f, f\rangle-\delta\langle\mathrm{AT}(1-\Pi) f, f\rangle+\delta\langle\mathrm{TA} f, f\rangle+2 \delta\langle\mathrm{AL} f, f\rangle$.

The first term in (17) satisfies the microscopic coercivity condition

$$
-\langle\mathrm{L} f, f\rangle \geq \lambda_{m}\|(1-\Pi) f\|^{2} .
$$

The second term in (17) is computed as follows. Using (5), we know that

$$
(\mathrm{T} \Pi)^{*} \mathrm{~T} \Pi f=-\Pi \mathrm{T}^{2} \Pi f=-\Theta M \Delta_{x} \rho_{f} .
$$

Solving $g=\operatorname{AT} \Pi f$ is equivalent to solving $\left(1+(\mathrm{T} \Pi)^{*} \mathrm{~T} \Pi\right) g=(\mathrm{T} \Pi)^{*} \mathrm{~T} \Pi f$, i.e.,

$$
v_{f}-\Theta \Delta_{x} v_{f}=-\Theta \Delta_{x} \rho_{f},
$$

where $g=v_{f} M$. Hence

$$
\langle\operatorname{AT} \Pi f, f\rangle=\int_{\mathbb{R}^{d}} v_{f} \rho_{f} d x .
$$

A direct application of the hypocoercivity approach of [10] to the whole space problem fails by lack of a macroscopic coercivity condition. Although the second term in (17) is not coercive, we observe that the last three terms in (17) can still be dominated by the first two for $\delta>0$, small enough.

- As in [10], we use the adjoint operators to compute

$$
\langle\mathrm{AT}(1-\Pi) f, f\rangle=-\left\langle(1-\Pi) f, \mathrm{TA}^{*} f\right\rangle .
$$

We observe that

$$
\mathrm{A}^{*} f=\mathrm{T} \Pi\left(1+(\mathrm{T} \Pi)^{*} \mathrm{~T} \Pi\right)^{-1} f=\mathrm{T}\left(1+(\mathrm{T} \Pi)^{*} \mathrm{~T} \Pi\right)^{-1} \Pi f=M \mathrm{~T} u_{f}
$$

where $u_{f}$ is the solution in $\mathrm{H}^{1}(d x)$ of

$$
u_{f}-\Theta \Delta_{x} u_{f}=\rho_{f} \text {. }
$$


With $K$ defined as a fourth order moment, we obtain that

$$
\mathrm{A}^{*} f=v M \cdot \nabla_{x} u_{f} \quad \text { and }\left\|\mathrm{TA}{ }^{*} f\right\|^{2} \leq K^{2}\left\|\nabla_{x}^{2} u_{f}\right\|_{\mathrm{L}^{2}(d x)}^{2}=K^{2}\left\|\Delta_{x} u_{f}\right\|_{\mathrm{L}^{2}(d x)}^{2} .
$$

On the other hand, we observe that $v_{f}=-\Theta \Delta u_{f}$ solves (18). Hence by multiplying (19) by $v_{f}=-\Theta \Delta u_{f}$ and integrating by parts, we know that

$$
\Theta\left\|\nabla_{x} u_{f}\right\|_{\mathrm{L}^{2}(d x)}^{2}+\Theta^{2}\left\|\Delta_{x} u_{f}\right\|_{\mathrm{L}^{2}(d x)}^{2}=\int_{\mathbb{R}^{d}} v_{f} \rho_{f} d x=\langle\operatorname{AT} \Pi f, f\rangle .
$$

Altogether we obtain that

$$
|\langle\mathrm{AT}(1-\Pi) f, f\rangle| \leq\|(1-\Pi) f\|\left\|\mathrm{TA}^{*} f\right\| \leq \frac{K}{\Theta}\|(1-\Pi) f\|\langle\mathrm{AT} \Pi f, f\rangle^{\frac{1}{2}} .
$$

- By (13), we have

$$
|\langle\mathrm{TA} f, f\rangle|=|\langle\mathrm{TA}(1-\Pi) f,(1-\Pi) f\rangle| \leq\|(1-\Pi) f\|^{2} .
$$

- It remains to estimate the last term on the right hand side of (17). Let us consider the solution $u_{f}$ of (19). If we multiply (18) by $u_{f}$ and integrate, we observe that

$$
\Theta\left\|\nabla_{x} u_{f}\right\|_{\mathrm{L}^{2}(d x)}^{2}=\int_{\mathbb{R}^{d}} u_{f} v_{f} d x \leq \int_{\mathbb{R}^{d}} u_{f} v_{f} d x+\int_{\mathbb{R}^{d}}\left|v_{f}\right|^{2} d x=\int_{\mathbb{R}^{d}} v_{f} \rho_{f} d x
$$

because $v_{f}=-\Theta \Delta u_{f}$, so that

$$
\left\|\mathrm{A}^{*} f\right\|^{2}=\Theta\left\|\nabla_{x} u_{f}\right\|_{\mathrm{L}^{2}(d x)}^{2} \leq\langle\mathrm{AT} f, f\rangle .
$$

In Case (a), we compute

$$
\langle\mathrm{AL} f, f\rangle=\left\langle\mathrm{L}(1-\Pi) f, \mathrm{~A}^{*} f\right\rangle=\iint_{\mathbb{R}^{d} \times \mathbb{R}^{d}} \nabla_{x} u_{f} \cdot \frac{\nabla_{v} M}{M}(1-\Pi) f d x d v .
$$

It follows from the Cauchy-Schwarz inequality that

$$
\int_{\mathbb{R}^{d}}\left|\nabla_{\nu} M\right||(1-\Pi) f| d \gamma \leq\left\|\nabla_{\nu} M\right\|_{\mathrm{L}^{2}(d \gamma)}\|(1-\Pi) f\|_{\mathrm{L}^{2}(d \gamma)}=\sqrt{d \theta}\|(1-\Pi) f\|_{\mathrm{L}^{2}(d \gamma)}
$$

and

$$
|\langle\mathrm{AL} f, f\rangle| \leq\left\|\nabla_{x} u_{f}\right\|_{\mathrm{L}^{2}(d x)}\left(\int_{\mathbb{R}^{d}}\left(\frac{1}{d} \int_{\mathbb{R}^{d}}\left|\nabla_{\nu} M\right||(1-\Pi) f| d \gamma\right)^{2} d x\right)^{\frac{1}{2}} .
$$

Altogether, we obtain that

$$
|\langle\mathrm{AL} f, f\rangle| \leq \sqrt{\frac{\theta}{\Theta}}\|(1-\Pi) f\|\langle\operatorname{AT} \Pi f, f\rangle^{\frac{1}{2}} .
$$

In Case (b), we use (3) to get that

$$
|\langle\mathrm{AL} f, f\rangle| \leq\|\mathrm{L} f\|\left\|\mathrm{A}^{*} f\right\| \leq 2 \bar{\sigma}\|(1-\Pi) f\|\left\|\mathrm{A}^{*} f\right\| \leq 2 \bar{\sigma}\|(1-\Pi) f\|\langle\mathrm{AT} \Pi f, f\rangle^{\frac{1}{2}} .
$$

In both cases, (a) and (b), the estimate can be written as

$$
|\langle\mathrm{AL} f, f\rangle| \leq 2 \bar{\sigma}\|(1-\Pi) f\|\langle\mathrm{AT} \Pi f, f\rangle^{\frac{1}{2}}
$$

with the convention that $\bar{\sigma}=\frac{1}{2} \sqrt{\theta / \Theta}$ in Case (a).

Summarizing, we know that

$$
-\frac{d}{d t} \mathrm{H}[f] \geq\left(\lambda_{m}-\delta\right) X^{2}+\delta Y^{2}+2 \delta \mathrm{b} X Y
$$


with $X:=\|(1-\Pi) f\|, Y:=\langle\text { AT } f, f\rangle^{1 / 2}$ and $\mathrm{b}:=\frac{K}{2 \Theta}+4 \bar{\sigma}$. The largest constant a $>0$ such that

$$
\left(\lambda_{m}-\delta\right) X^{2}+\delta Y^{2}+2 \delta \mathrm{b} X Y \geq \mathrm{a}\left(X^{2}+2 Y^{2}\right)
$$

holds for any $X, Y \in \mathbb{R}$ is given by the conditions

$$
\mathrm{a}<\lambda_{m}-\delta, \quad 2 \mathrm{a}<\delta<1, \quad \delta^{2} \mathrm{~b}^{2}-\left(\lambda_{m}-\delta-\mathrm{a}\right)(\delta-2 \mathrm{a}) \leq 0
$$

and it is easy to check that there exists a positive solution if $\delta>0$ is small enough. We can for instance choose

$$
\delta=\frac{4 \min \left\{1, \lambda_{m}\right\}}{8 \mathrm{~b}^{2}+5} \text { and } \mathrm{a}=\frac{\delta}{4} .
$$

Altogether we obtain that

$$
-\frac{d}{d t} \mathrm{H}[f] \geq \mathrm{a}\left(\|(1-\Pi) f\|^{2}+2\langle\text { AT } \Pi f, f\rangle\right) .
$$

Using (19) and (20), we control $\|\Pi f\|^{2}=\left\|\rho_{f}\right\|_{\mathrm{L}^{2}(d x)}^{2}$ by $\langle$ AT $\Pi f, f\rangle$ according to

$$
\begin{aligned}
&\|\Pi f\|^{2}=\left\|u_{f}\right\|_{\mathrm{L}^{2}(d x)}^{2}+2 \Theta\left\|\nabla_{x} u_{f}\right\|_{\mathrm{L}^{2}(d x)}^{2}+\Theta^{2}\left\|\Delta_{x} u_{f}\right\|_{\mathrm{L}^{2}(d x)}^{2} \\
& \leq\left\|u_{f}\right\|_{\mathrm{L}^{2}(d x)}^{2}+2\langle\operatorname{AT} \Pi f, f\rangle .
\end{aligned}
$$

We observe that

$$
\left\|u_{f}(t, \cdot)\right\|_{\mathrm{L}^{1}(d x)}=\left\|\rho_{f}(t, \cdot)\right\|_{\mathrm{L}^{1}(d x)}=\left\|f_{0}\right\|_{\mathrm{L}^{1}(d x d v)}, \quad\left\|\nabla_{x} u_{f}\right\|_{\mathrm{L}^{2}(d x)}^{2} \leq \frac{1}{\Theta}\langle\operatorname{AT} \Pi f, f\rangle
$$

for any $t \geq 0$ and use (16) with $u=u_{f}$ to get

$$
\|\Pi f\|^{2} \leq \Phi^{-1}(2\langle\text { АТ } \Pi f, f\rangle)
$$

with

$$
\Phi^{-1}(y):=y+\left(\frac{y}{c}\right)^{\frac{d}{d+2}} \quad \forall y \geq 0, \quad \text { and } \quad c=2 \Theta \mathscr{C}_{\text {Nash }}^{-1-\frac{2}{d}}\left\|f_{0}\right\|_{\mathrm{L}^{1}(d x d v)}^{-\frac{4}{d}} .
$$

The function $\Phi:[0, \infty) \rightarrow[0, \infty)$ satisfies $\Phi(0)=0$ and $0<\Phi^{\prime}<1$, so that

$$
\|(1-\Pi) f\|^{2}+2\langle\text { AT } f, f\rangle \geq \Phi\left(\|f\|^{2}\right) \geq \Phi\left(\frac{2}{1+\delta} \mathrm{H}[f]\right)
$$

where the last inequality holds as a consequence of (14) and of Plancherel's formula. From

we read that

$$
z=\Phi^{-1}(y) \geq\left(\frac{y}{\mathrm{c}}\right)^{\frac{d}{d+2}}
$$

$$
y=\Phi(z) \leq c z^{1+\frac{2}{d}}
$$

and deduce the entropy decay inequality

$$
-\frac{d}{d t} \mathrm{H}[f] \geq \operatorname{ac}\left(\frac{2}{1+\delta} \mathrm{H}[f]\right)^{1+\frac{2}{d}} .
$$

A simple integration from 0 to $t$ shows that

$$
\mathrm{H}[f] \leq\left(\mathrm{H}\left[f_{0}\right]^{-\frac{2}{d}}+\frac{2 \mathrm{ac}}{d}\left(\frac{2}{1+\delta}\right)^{1+\frac{2}{d}} t\right)^{-\frac{d}{2}} .
$$

The result of Theorem 8 follows from elementary considerations, using the fact that $\mathrm{c}$ is proportional to $\left\|f_{0}\right\|_{\mathrm{L}^{1}(d x d v)}^{-4 / d}$. 
5.2. Improved decay rate for zero average solutions. - Let us prove Theorem 2. We replace Nash's inequality (16) by an analysis based on some Fourier methods.

- Step 1: Decay of the average in space by a factorization argument.- We define

$$
f_{\bullet}(t, v):=\int_{\mathbb{R}^{d}} f(t, x, v) d x
$$

and observe that $f_{\bullet}$ solves

$$
\partial_{t} f_{\bullet}=\mathrm{L} f_{\bullet} .
$$

As a consequence, we have that $0=\int_{\mathbb{R}^{d}} f_{\bullet}(t, v) d v=\int_{\mathbb{R}^{d}} \frac{f_{\cdot(t, v)}}{M} M d v$. From the microscopic coercivity property $(\mathrm{P})$, we deduce that

$$
\left\|f_{\bullet}(t, \cdot)\right\|_{\mathrm{L}^{2}(d \gamma)}^{2}=\int_{\mathbb{R}^{d}}\left|\frac{f_{\bullet}(t, v)}{M}\right|^{2} M d v \leq\left\|f_{\bullet}(0, \cdot)\right\|_{\mathrm{L}^{2}(d \gamma)}^{2} e^{-\lambda_{m} t} \quad \forall t \geq 0 .
$$

With $k \in(d, \infty)$, Theorem 5 applies like in the proof of Theorem 6 or in [26]. We observe that $\left\|f_{\bullet}(0, \cdot)\right\|_{\mathrm{L}^{2}\left(|v|^{2} d \gamma_{k}\right)}=\left\|f_{0}\right\|_{\mathrm{L}^{2}\left(|v|^{2} d \gamma_{k} ; \mathrm{L}^{1}(d x)\right)}$. For some positive constants $C$ and $\lambda$, we get that

$$
\left\|f_{\bullet}(t, \cdot)\right\|_{\mathrm{L}^{2}\left(|v|^{2} d \gamma_{k}\right)}^{2} \leq C\left\|f_{0}\right\|_{\mathrm{L}^{2}\left(|v|^{2} d \gamma_{k} ; \mathrm{L}^{1}(d x)\right)}^{2} e^{-\lambda t}, \quad \forall t \geq 0 .
$$

Notice that the case $k=\infty$, i.e, $d \gamma_{\infty}=d \gamma=M^{-1} d v$, could also be considered in Theorem 2, up to additional conditions on $M$, satisfied for instance if $M$ is a Gaussian. Then (23) holds with $|v|^{2} d \gamma_{k}$ replaced by $|v|^{2} d \gamma$, as in [11, Ineq. (4)]: for instance, in the Gaussian case, we know that $2(d+2) \int_{\mathbb{R}^{d}}\left|\nabla f_{\bullet}\right|^{2} d \gamma \geq \int_{\mathbb{R}^{d}}|v|^{2}\left|f_{\bullet}\right|^{2} d \gamma$.

- Step 2: Improved decay of $f$.- Let us define $g:=f-f_{\bullet} \varphi$, where $\varphi$ is a given positive function such that $\hat{\varphi}(0)=\int \varphi d x=1$, for instance

$$
\varphi(x):=(2 \pi)^{-d / 2} e^{-|x|^{2} / 2} \quad \forall x \in \mathbb{R}^{d} .
$$

Since $\partial_{t} f_{\bullet}=\mathrm{L} f_{\bullet}$, the Fourier transform $\hat{g}$ solves

$$
\partial_{t} \hat{g}+\mathrm{T} \hat{g}=\mathrm{L} \hat{g}-f_{\bullet} \mathrm{T} \hat{\varphi},
$$

where $\mathrm{T} \hat{\varphi}=i(\nu \cdot \xi) \hat{\varphi}$. Using Duhamel's formula

$$
\hat{g}=e^{i(\mathrm{~L}-\mathrm{T}) t} \hat{g}_{0}+\int_{0}^{t} e^{i(\mathrm{~L}-\mathrm{T})(t-s)}\left(-f_{\bullet}(s, v) \mathrm{T} \hat{\varphi}(\xi)\right) d s,
$$

Theorem 4 and Theorem 5, for some generic constant $C>0$ which will change from line to line, we get

$$
\begin{aligned}
\|\hat{g}(t, \xi, \cdot)\|_{L^{2}\left(d \gamma_{k}\right)} \leq C e^{-\frac{1}{2} \mu_{\xi} t} & \left\|\hat{g}_{0}(\xi, \cdot)\right\|_{L^{2}\left(d \gamma_{k}\right)} \\
& +C \int_{0}^{t} e^{-\frac{\mu_{\xi}}{2}(t-s)}\left\|f_{\bullet}(s, \cdot)\right\|_{L^{2}\left(|v|^{2} d \gamma_{k}\right)}|\xi||\hat{\varphi}(\xi)| d s .
\end{aligned}
$$

The key observation is $\hat{g}_{0}(0, v)=0$, so that $\hat{g}_{0}(\xi, v)=\int_{0}^{|\xi|} \frac{\xi}{|\xi|} \cdot \nabla_{\xi} \hat{g}_{0}\left(\eta \frac{\xi}{|\xi|}, v\right) d \eta$ yields

$$
\left|\hat{g}_{0}(\xi, v)\right| \leq|\xi|\left\|\nabla_{\xi} \hat{g}_{0}(\cdot, v)\right\|_{L^{\infty}(d v)} \leq|\xi|\left\|g_{0}(\cdot, v)\right\|_{L^{1}(|x| d x)} \quad \forall(\xi, v) \in \mathbb{R}^{d} \times \mathbb{R}^{d} .
$$


We know from (15) that $\mu_{\xi}=\Lambda|\xi|^{2} /\left(1+|\xi|^{2}\right)$. The first term of the r.h.s. of (24) can therefore be estimated for any $t \geq 1$ by

$$
\begin{array}{r}
\left(\int_{|\xi| \leq 1} \int_{\mathbb{R}^{d}}\left|e^{i(\mathrm{~L}-\mathrm{T}) t} \hat{g}_{0}\right|^{2} d \gamma_{k} d \xi\right)^{1 / 2} \leq\left(\int_{\mathbb{R}^{d}}|\xi|^{2} e^{-\frac{\Lambda}{2}|\xi|^{2} t} d \xi\right)^{1 / 2}\left\|g_{0}\right\|_{\mathrm{L}^{2}\left(d \gamma_{k} ; \mathrm{L}^{1}(|x| d x)\right)} \\
\leq \frac{C}{(1+t)^{1+\frac{d}{2}}}\left\|g_{0}\right\|_{\mathrm{L}^{2}\left(d \gamma_{k} ; \mathrm{L}^{1}(|x| d x)\right)},
\end{array}
$$

which is the leading order term as $t \rightarrow \infty$, and

$$
\int_{|\xi|>1} e^{-\mu_{\xi} t}\left\|\hat{g}_{0}(\xi, \cdot)\right\|_{\mathrm{L}^{2}\left(d \gamma_{k}\right)}^{2} d \xi \leq C e^{-\frac{\Lambda}{2} t}\left\|g_{0}\right\|_{\mathrm{L}^{2}\left(d x d \gamma_{k}\right)}^{2},
$$

using the fact that $\mu_{\xi} \geq \Lambda / 2$ when $|\xi| \geq 1$ and Plancherel's formula.

Using (23), the second term of the r.h.s. of (24) is estimated by

$$
\begin{aligned}
\int_{\mathbb{R}^{d}}\left(\int_{0}^{t} e^{-\frac{\mu_{\xi}}{2}(t-s)}\left\|f_{\bullet}(s, \cdot)\right\|_{\mathrm{L}^{2}\left(|\nu|^{2} d \gamma_{k}\right)}|\xi||\hat{\varphi}(\xi)| d s\right)^{2} d \xi \\
\quad \leq C\left\|f_{0}\right\|_{\mathrm{L}^{2}\left(|\nu|^{2} d \gamma_{k} ; \mathrm{L}^{1}(d x)\right)}^{2} \int_{\mathbb{R}^{d}}|\xi|^{2}|\hat{\varphi}(\xi)|^{2}\left(\int_{0}^{t} e^{-\frac{\mu_{\xi}}{2}(t-s)} e^{-\frac{\lambda}{2} s} d s\right)^{2} d \xi .
\end{aligned}
$$

On the one hand, we use the Cauchy-Schwarz inequality to get

$$
\begin{aligned}
\int_{|\xi| \leq 1} & |\xi|^{2}|\hat{\varphi}(\xi)|^{2}\left(\int_{0}^{t} e^{-\frac{\mu_{\xi}}{2}(t-s)} e^{-\frac{\lambda}{2} s} d s\right)^{2} d \xi \\
& \leq\|\varphi\|_{\mathrm{L}^{1}(d x)}^{2} \int_{|\xi| \leq 1}|\xi|^{2}\left(\int_{0}^{t} e^{-\mu_{\xi}(t-s)} e^{-\frac{\lambda}{2} s} d s\right)\left(\int_{0}^{t} e^{-\frac{\lambda}{2} s} d s\right) d \xi \\
& \leq \frac{2}{\lambda}\|\varphi\|_{\mathrm{L}^{1}(d x)}^{2} \int_{0}^{t}\left(\int_{|\xi| \leq 1}|\xi|^{2} e^{-\frac{\Lambda}{2}|\xi|^{2}(t-s)} d \xi\right) e^{-\frac{\lambda}{2} s} d s \leq C_{1} t^{-\frac{d}{2}-1}+C_{2} e^{-\frac{\lambda}{4} t},
\end{aligned}
$$

where the last inequality is obtained by splitting the integral in $s$ on $(0, t / 2)$ and $(t / 2, t)$. On the other hand, using $\mu_{\xi} \geq \Lambda / 2$ when $|\xi| \geq 1$, we obtain

$$
\int_{|\xi| \geq 1}|\xi|^{2}|\hat{\varphi}(\xi)|^{2}\left(\int_{0}^{t} e^{-\frac{\mu_{\xi}}{2}(t-s)} e^{-\frac{\lambda}{2} s} d s\right)^{2} d \xi \leq t^{2} e^{-\min \{\Lambda / 2, \lambda\} t}\|\nabla \varphi\|_{\mathrm{L}^{2}(d x)}^{2} .
$$

By collecting all terms, we deduce that $\|g(t, \cdot, \cdot)\|_{\mathrm{L}^{2}\left(d x d \gamma_{k}\right)}^{2}$ is bounded by

$$
C\left(\left\|g_{0}\right\|_{\mathrm{L}^{2}\left(d \gamma_{k} ; \mathrm{L}^{1}(|x| d x)\right)}^{2}+\left\|f_{0}\right\|_{\mathrm{L}^{2}\left(\left.|| \nu\right|^{2} d \gamma_{k} ; \mathrm{L}^{1}(d x)\right)}^{2}\right)(1+t)^{-\left(1+\frac{d}{2}\right)},
$$

for some constant $C>0$. Recalling that $f=g+f_{\bullet} \varphi$, the proof of Theorem 2 is completed using (23). 
A

\section{An explicit computation of Green's function for the kinetic Fokker-Planck equation and consequences}

In the whole space case, when $M$ is the normalized Gaussian function, let us consider the kinetic Fokker-Planck equation of Case (a)

$$
\partial_{t} f+v \cdot \nabla_{x} f=\nabla_{v} \cdot\left(v f+\nabla_{v} f\right)
$$

on $(0, \infty) \times \mathbb{R}^{d} \times \mathbb{R}^{d} \ni(t, x, v)$. The characteristics associated with the equations

$$
\frac{d x}{d t}=v, \quad \frac{d v}{d t}=-v
$$

suggest to change variables and consider the distribution function $g$ such that

$$
f(t, x, v)=e^{d t} g\left(t, x+\left(1-e^{t}\right) v, e^{t} v\right) \quad \forall(t, x, v) \in(0, \infty) \times \mathbb{R}^{d} \times \mathbb{R}^{d} .
$$

The kinetic Fokker-Planck equation is changed into a heat equation in both variables $x$ and $v$ with $t$ dependent coefficients, which can be written as

$$
\partial_{t} g=\nabla \cdot \dot{D} \nabla g
$$

where $\nabla g=\left(\nabla_{\nu} g, \nabla_{x} g\right)$ and $\dot{\mathscr{D}}$ is the $t$-derivative of the bloc-matrix

$$
\mathscr{D}=\frac{1}{2}\left(\begin{array}{ll}
\text { a Id } & \text { bId } \\
\text { bId } & \text { cId }
\end{array}\right)
$$

with $\mathrm{a}=e^{2 t}-1, \mathrm{~b}=2 e^{t}-1-e^{2 t}$, and $\mathrm{c}=e^{2 t}-4 e^{t}+2 t+3$. Here Id is the identity matrix on $\mathbb{R}^{d}$. We observe that $\dot{\mathscr{D}}$ is degenerate: it is nonnegative but its lowest eigenvalue is 0 . However, the change of variables allows the computation of a Green function.

Lemma 9. - The Green function of (26) is given by

$$
G(t, x, v)=\frac{1}{\left(2 \pi\left(\mathrm{ac}-\mathrm{b}^{2}\right)\right)^{d}} e^{-\frac{\mathrm{a}|x|^{2}-2 \mathrm{~b} x \cdot v+\mathrm{c}|\nu|^{2}}{2\left(\mathrm{ac}-\mathrm{b}^{2}\right)}}, \quad(t, x, v) \in(0, \infty) \times \mathbb{R}^{d} \times \mathbb{R}^{d} .
$$

The method is standard and goes back to $[25]$ (also see $[22,20]$ and $[32,5])$.

Proof. - By a Fourier transformation in $x$ and $v$, with associated variables $\xi$ and $\eta$, we find that

$$
\begin{aligned}
\log C-\log \hat{G}(t, \xi, \eta)=(\eta, \xi) \cdot \mathscr{D}(\eta, \xi)=\frac{1}{2}\left(\mathrm{a}|\eta|^{2}+2 \mathrm{~b} \eta \cdot \xi+\mathrm{c}|\xi|^{2}\right) & \\
& =\frac{1}{2} \mathrm{a}\left|\eta+\frac{\mathrm{b}}{\mathrm{a}} \xi\right|^{2}+\frac{1}{2} \mathrm{~A}|\xi|^{2}, \quad \mathrm{~A}=\mathrm{c}-\frac{\mathrm{b}^{2}}{\mathrm{a}}
\end{aligned}
$$

for some constant $C>0$ which is determined by the mass normalization condition $\|G(t, \cdot \cdot)\|_{\mathrm{L}^{1}\left(\mathbb{R}^{d} \times \mathbb{R}^{d}\right)}=1$. Let us take the inverse Fourier transform with respect to $\eta$,

$$
\begin{aligned}
(2 \pi)^{-d} \int_{\mathbb{R}^{d}} e^{i v \cdot \eta} \hat{G}(t, \xi, \eta) d \eta=\frac{C}{(2 \pi \mathrm{a})^{d}} e^{-\frac{|\nu|^{2}}{2 \mathrm{a}}-i \frac{\mathrm{b}}{\mathrm{a}} v \cdot \xi} e^{-\frac{1}{2} \mathrm{~A}|\xi|^{2}} \\
=\frac{C}{(2 \pi \mathrm{a})^{d}} e^{-\frac{|\nu|^{2}}{2 \mathrm{a}}} e^{-\frac{1}{2} \mathrm{~A}\left|\xi+i \frac{\mathrm{b}}{\mathrm{a} \mathrm{A}} \nu\right|^{2}-\frac{\mathrm{b}^{2}}{2 \mathrm{a}^{2} \mathrm{~A}}|\nu|^{2}},
\end{aligned}
$$


and then the inverse Fourier transform with respect to $\xi$, so that we obtain

$$
G(t, x, v)=\frac{C}{(2 \pi \mathrm{a})^{d}(2 \pi \mathrm{A})^{d}} e^{-\left(1+\frac{\mathrm{b}^{2}}{\mathrm{aA}}\right) \frac{|v|^{2}}{2 \mathrm{a}}} e^{-\frac{|x|^{2}}{2 \mathrm{~A}}} e^{\frac{\mathrm{b}}{\mathrm{aA}} x \cdot v}=\frac{C}{\left(4 \pi^{2} \mathrm{a} \mathrm{A}\right)^{d}} e^{-\frac{1}{2 \mathrm{~A}}\left|x-\frac{\mathrm{b}}{\mathrm{a}} \nu\right|^{2}} e^{-\frac{|v|^{2}}{2 \mathrm{a}}} .
$$

It is easy to check that $C=(2 \pi)^{d}$.

Let us consider a solution $g$ of (26) with initial datum $g_{0} \in \mathrm{L}^{1}\left(\mathbb{R}^{d} \times \mathbb{R}^{d}\right)$. From the representation

we obtain the estimate

$$
g(t, \cdot, \cdot)=G(t, \cdot, \cdot) * x, v g_{0}
$$

$$
\begin{aligned}
\|g(t, \cdot, \cdot)\|_{L^{\infty}\left(\mathbb{R}^{d} \times \mathbb{R}^{d}\right)} \leq\|G(t, \cdot, \cdot)\|_{\mathrm{L}^{\infty}\left(\mathbb{R}^{d} \times \mathbb{R}^{d}\right)}\left\|g_{0}\right\|_{\mathrm{L}^{1}\left(\mathbb{R}^{d} \times \mathbb{R}^{d}\right)} & \\
= & {\left[4 \pi\left(e^{t}-1\right)\left((t-2) e^{t}+t+2\right)\right]^{-d} \sim t^{-d} e^{-d t} }
\end{aligned}
$$

as $t \rightarrow \infty$. As a consequence, we obtain that the solution of (25) with a nonnegative initial datum $f_{0}$ satisfies

$$
\|f(t, \cdot, \cdot)\|_{\mathrm{L}^{\infty}\left(\mathbb{R}^{d} \times \mathbb{R}^{d}\right)}=\frac{\left\|f_{0}\right\|_{\mathrm{L}^{1}\left(\mathbb{R}^{d} \times \mathbb{R}^{d}\right)}}{(4 \pi t)^{d}}(1+o(1)) \quad \text { as } \quad t \rightarrow \infty .
$$

Using the simple Hölder interpolation inequality

$$
\|f\|_{\mathrm{L}^{p}\left(\mathbb{R}^{d} \times \mathbb{R}^{d}\right)} \leq\|f\|_{\mathrm{L}^{1}\left(\mathbb{R}^{d} \times \mathbb{R}^{d}\right)}^{1 / p}\|f\|_{\mathrm{L}^{\infty}\left(\mathbb{R}^{d} \times \mathbb{R}^{d}\right)}^{1-1 / p},
$$

we obtain the following decay result.

Corollary 10. - If $f$ is a solution of (25) with a nonnegative initial datum $f_{0} \in$ $\mathrm{L}^{1}\left(\mathbb{R}^{d} \times \mathbb{R}^{d}\right)$, then for any $p \in(1, \infty]$ we have the decay estimate

$$
\|f(t, \cdot, \cdot)\|_{\mathrm{L}^{p}\left(\mathbb{R}^{d} \times \mathbb{R}^{d}\right)}=\frac{\left\|f_{0}\right\|_{\mathrm{L}^{1}\left(\mathbb{R}^{d} \times \mathbb{R}^{d}\right)}}{(4 \pi t)^{d(1-1 / p)}}(1+o(1)) \quad \text { as } \quad t \rightarrow \infty .
$$

By taking $f_{0}(x, v)=G(1, x, v)$, it is moreover straightforward to check that this estimate is optimal. With $p=2$, this also proves that the decay rate obtained in Theorem 1 for the Fokker-Planck operator, i.e., Case (a), is the optimal one.

\section{B}

\section{Consistency with the decay rates of the heat equation}

In the whole space case, the abstract approach of [10] is inspired by the diffusion limit of (1). We consider the scaled equation

$$
\varepsilon \frac{d F}{d t}+\mathrm{T} F=\frac{1}{\varepsilon} \mathrm{L} F
$$

which formally corresponds to a parabolic rescaling given by $t \mapsto \varepsilon^{2} t$ and $x \mapsto \varepsilon x$, and investigate the limit as $\varepsilon \rightarrow 0_{+}$. Let us check that the rates are asymptotically independent of $\varepsilon$ and consistent with those of the heat equation.

B.1. Mode-by-mode hypocoercivity. - It is straightforward to check that in the estimate (12) for $\lambda$, the gap constant $\lambda_{m}$ has to be replaced by $\lambda_{m} / \varepsilon$ while, with the 
notations of Theorem 3, $C_{M}$ can be replaced by $C_{M} / \varepsilon$ for $\varepsilon<1$. In the asymptotic regime as $\varepsilon \rightarrow 0_{+}$, we obtain that

$$
\varepsilon \frac{d}{d t} \mathrm{H}[F] \leq-\mathrm{D}[F] \leq-\frac{\lambda_{M}}{3\left(1+\lambda_{M}\right)} \frac{\lambda_{m} \lambda_{M} \varepsilon}{\left(1+\lambda_{M}\right) C_{M}^{2}} \mathrm{D}[F]
$$

which proves that the estimate of Theorem 3 becomes

$$
\lambda \geq \frac{\lambda_{m} \lambda_{M}^{2}}{3\left(1+\lambda_{M}\right)^{2} C_{M}^{2}}
$$

for $\varepsilon>0$, small enough. We observe that this rate is independent of $\varepsilon$.

B.2. Decay rates based on Nash's inequality in the whole space case. - In the proof of Theorem $8, \bar{\sigma}$ has to be replaced by $\bar{\sigma} / \varepsilon$ and in the limit as $\varepsilon \rightarrow 0_{+}$, we get that $\mathrm{b} \sim 4 \bar{\sigma} / \varepsilon$ and (21) is satisfied with $4 \mathrm{a}=\delta \sim \frac{\lambda_{m}}{4 \bar{\sigma}^{2}} \varepsilon$. Hence (22) becomes

$$
-\frac{d}{d t} \mathrm{H}[f] \geq \frac{\lambda_{m}}{4 \bar{\sigma}^{2}} \mathrm{c}\left(\frac{2}{1+\delta} \mathrm{H}[f]\right)^{1+\frac{2}{d}},
$$

which again gives a rate of decay which is independent of $\varepsilon$. The algebraic decay rate in Theorem 1 or Theorem 8 is the one of the heat equation on $\mathbb{R}^{d}$ and it is independent of $\varepsilon$ in the limit as $\varepsilon \rightarrow 0_{+}$.

B.3. Decay rates in the whole space case for zero-average distribution functions. - The improved rate of Theorem 2 is consistent with a parabolic rescaling: if $f$ solves (1), then $f^{\varepsilon}(t, x, v)=\varepsilon^{-d} f\left(\varepsilon^{-2} t, \varepsilon^{-1} x, v\right)$ solves (27). With the notations of Section 5.2, let $g^{\varepsilon}=f^{\varepsilon}-f_{\bullet}^{\varepsilon} \varphi(\cdot / \varepsilon)$, with $\varphi^{\varepsilon}=\varepsilon^{-d} \varphi(\cdot / \varepsilon)$. The Fourier transform of $g^{\varepsilon}$ solves

$$
\varepsilon^{2} \partial_{t} \hat{g}^{\varepsilon}+\varepsilon \mathrm{T} \hat{g}^{\varepsilon}=\mathrm{L} \hat{g}^{\varepsilon}-\varepsilon f_{\bullet}^{\varepsilon} \mathrm{T} \hat{\varphi}^{\varepsilon} .
$$

The decay rate $\lambda$ in (23) becomes $\lambda / \varepsilon^{2}$ and the decay rate of the semi-group generated by $\mathrm{L}-\varepsilon \mathrm{T}$ is, with the notations of Theorem $4, \mu_{\varepsilon \xi}$. Moreover, $\Lambda$ in (15) is given by $\Lambda=\frac{1}{3} \min \{1,1 / \Theta\}$ for any $\varepsilon>0$, small enough. Duhamel's formula (24) has to be replaced by

$$
\begin{aligned}
\left\|\hat{g}^{\varepsilon}(t, \xi, \cdot)\right\|_{\mathrm{L}^{2}\left(d \gamma_{k}\right)} \leq C e^{-\frac{\mu_{\varepsilon \xi}}{2 \varepsilon^{2}} t}\left\|\hat{g}_{0}^{\varepsilon}(\xi, \cdot)\right\|_{\mathrm{L}^{2}\left(d \gamma_{k}\right)} & \\
& +C \int_{0}^{t} e^{-\frac{\mu_{\varepsilon \xi}}{2 \varepsilon^{2}}(t-s)}\left\|f_{\bullet}^{\varepsilon}(s, \cdot)\right\|_{\mathrm{L}^{2}\left(|\nu|^{2} d \gamma_{k}\right)}|\varepsilon \xi||\hat{\varphi}(\varepsilon \xi)| d s .
\end{aligned}
$$

Using $\lim _{\varepsilon \rightarrow 0_{+}} \frac{\mu_{\varepsilon \xi}}{\varepsilon^{2}}=\lim _{\varepsilon \rightarrow 0_{+}} \frac{\Lambda|\xi|^{2}}{1+\varepsilon^{2}|\xi|^{2}}=\Lambda|\xi|^{2}$, a computation similar to the one of Section 5.2 shows that the first term of the r.h.s. is estimated by

$$
\begin{aligned}
& \int_{\mathbb{R}^{d}} e^{-\frac{\mu_{\varepsilon \xi}}{\varepsilon^{2}} t}\left\|\hat{g}_{0}^{\varepsilon}(\xi, \cdot)\right\|_{\mathrm{L}^{2}\left(d \gamma_{k}\right)}^{2} d \xi \\
& =\int_{|\xi| \leq \frac{1}{\varepsilon}} e^{-\frac{\mu_{\varepsilon \xi} \xi}{\varepsilon^{2}} t}\left\|\hat{g}_{0}^{\varepsilon}(\xi, \cdot)\right\|_{\mathrm{L}^{2}\left(d \gamma_{k}\right)}^{2} d \xi+\int_{|\xi|>\frac{1}{\varepsilon}} e^{-\frac{\mu_{\varepsilon \xi}}{\varepsilon^{2}} t}\left\|\hat{g}_{0}^{\varepsilon}(\xi, \cdot)\right\|_{\mathrm{L}^{2}\left(d \gamma_{k}\right)}^{2} d \xi \\
& \leq\left\|g_{0}^{\varepsilon}\right\|_{\mathrm{L}^{2}\left(d \gamma_{k} ; \mathrm{L}^{1}(|x| d x)\right)}^{2} \int_{\mathbb{R}^{d}}|\xi|^{2} e^{-\frac{\Lambda}{2}|\xi|^{2} t} d \xi+\left\|g_{0}^{\varepsilon}\right\|_{\mathrm{L}^{2}\left(d x d \gamma_{k}\right)}^{2} e^{-\frac{\Lambda}{2} \frac{t}{\varepsilon^{2}}},
\end{aligned}
$$


while the second term is bounded by

$$
\begin{array}{r}
\left.\left\|f_{\bullet}^{\varepsilon}(t=0, \cdot)\right\|_{\mathrm{L}^{2}\left(|v|^{2} d \gamma_{k}\right)}^{2} \int_{\mathbb{R}^{d}}|\varepsilon| \xi\right|^{2}|\hat{\varphi}(\varepsilon \xi)|^{2}\left(\int_{0}^{\varepsilon^{-2} t} e^{-\frac{1}{2} \mu_{\varepsilon \xi}\left(\varepsilon^{-2} t-s\right)} e^{-\frac{1}{2} \lambda s} d s\right)^{2} d \xi \\
\leq\left\|f_{0}\right\|_{\mathrm{L}^{2}\left(|v|^{2} d \gamma_{k} ; \mathrm{L}^{1}(d x)\right)}^{2}\left(C_{1} \frac{\varepsilon^{d+1}}{t^{\frac{d}{2}+1}}+\frac{C_{2}}{\varepsilon^{3}} e^{-\min \left\{\frac{\Lambda}{2}, \lambda\right\} \frac{t}{\varepsilon^{2}}}\right) .
\end{array}
$$

By collecting all terms and using Plancherel's formula, we conclude that the rate of convergence of Theorem 2 applied to the solution of (27) is independent of $\varepsilon$. We also notice that the scaled spatial density $\rho_{f^{\varepsilon}}=\int_{\mathbb{R}^{d}} f^{\varepsilon} d v$ satisfies

$$
\left\|\rho_{f^{\varepsilon}}(t, \cdot)\right\|_{\mathrm{L}^{2}(d x)}^{2} \leq \frac{\mathscr{C}_{0}}{(1+t)^{1+\frac{d}{2}}} \quad \forall t \geq 0
$$

for some positive constant $\mathscr{C}_{0}$ which depends on $f_{0}$ but is independent of $\varepsilon$. This is the decay of the heat equation with an initial datum of zero average.

\section{Acknowledgments}

This work has been partially supported by the Projects EFI (E.B., J.D., ANR-17-CE400030), Kibord (E.B., J.D., ANR-13-BS01-0004) and STAB (J.D., ANR-12-BS01-0019) of the French National Research Agency (ANR). Support by the Austrian Science Foundation (grants no. F65 and W1245) is acknowledged by C.S. Moreover C.M. and C.S. are very grateful for the hospitality at Université Paris-Dauphine.

(c) 2017 by the authors. This paper may be reproduced, in its entirety, for non-commercial purposes.

\section{References}

[1] Achleitner, F., Arnold, A., And Carlen, E. A. On linear hypocoercive BGK models. In From Particle Systems to Partial Differential Equations III. Springer, 2016, pp. 1-37.

[2] Bakry, D., Barthe, F., Cattiaux, P., And Guillin, A. A simple proof of the Poincaré inequality for a large class of probability measures including the log-concave case. Electronic Communications in Probability 13 (2008), 60-66.

[3] Bartier, J.-P., Blanchet, A., Dolbeault, J., And Escobedo, M. Improved intermediate asymptotics for the heat equation. Applied Mathematics Letters 24, 1 (2011), $76-81$.

[4] Bhatnagar, P. L., Gross, E. P., AND Krook, M. A model for collision processes in gases. i. small amplitude processes in charged and neutral one-component systems. Physical review 94, 3 (1954), 511.

[5] Bouchut, F. Existence and uniqueness of a global smooth solution for the VlasovPoisson-Fokker-Planck system in three dimensions. J. Funct. Anal. 111, 1 (1993), 239258.

[6] Bouin, E., Hoffmann, F., AND Mounot, C. Exponential decay to equilibrium for a fibre lay-down process on a moving conveyor belt. to appear in SIAM Journal on Mathematical Analysis, ArXiv e-prints (May 2016).

[7] CÁceres, M. J., CARrillo, J. A., ANd Goudon, T. Equilibration rate for the linear inhomogeneous relaxation-time Boltzmann equation for charged particles. Comm. Partial Differential Equations 28, 5-6 (2003), 969-989. 
[8] Degond, P., Goudon, T., And Poupaud, F. Diffusion limit for nonhomogeneous and non-microreversible processes. Indiana Univ. Math. J. 49 (2000), 1175-1198.

[9] Dolbeault, J., Mouhot, C., And Schmeiser, C. Hypocoercivity for kinetic equations with linear relaxation terms. Comptes Rendus Mathématique 347, 9-10 (2009), $511-516$.

[10] Dolbeault, J., Mouhot, C., And Schmeiser, C. Hypocoercivity for linear kinetic equations conserving mass. Trans. AMS 367 (2015), 3807-3828.

[11] Dolbeault, J., And Volzone, B. Improved Poincaré inequalities. Nonlinear Analysis: Theory, Methods \& Applications 75, 16 (2012), 5985 - 6001.

[12] DUAN, R. Hypocoercivity of linear degenerately dissipative kinetic equations. Nonlinearity 24, 8 (2011), 2165-2189.

[13] ECKMAnn, J.-P., AND HAIRER, M. Spectral properties of hypoelliptic operators. Comm. Math. Phys. 235, 2 (2003), 233-253.

[14] Evans, J. Hypocoercivity in Phi-entropy for the linear Boltzmann equation on the torus. ArXiv e-prints (Feb. 2017).

[15] GLAssey, R. T. The Cauchy problem in kinetic theory. Society for Industrial and Applied Mathematics (SIAM), Philadelphia, PA, 1996.

[16] Gualdani, M. P., Mischler, S., And Mouhot, C. Factorization for non-symmetric operators and exponential H-theorem. ArXiv e-prints, to appear as a Mémoire de la Société Mathématique de France (118 pages) (June 2010).

[17] Han-Kwan, D., And LÉAutaud, M. Geometric analysis of the linear Boltzmann equation I. Trend to equilibrium. Ann. PDE 1, 1 (2015), Art. 3, 84.

[18] HÉraU, F. Hypocoercivity and exponential time decay for the linear inhomogeneous relaxation Boltzmann equation. Asymptot. Anal. 46, 3-4 (2006), 349-359.

[19] HÉrAU, F., AND Nier, F. Isotropic hypoellipticity and trend to equilibrium for the Fokker-Planck equation with a high-degree potential. Arch. Ration. Mech. Anal. 171, 2 (2004), 151-218.

[20] Hörmander, L. Hypoelliptic second order differential equations. Acta Math. 119 (1967), 147-171.

[21] Iacobucci, A., Olla, S., AND Stoltz, G. Convergence rates for nonequilibrium Langevin dynamics. ArXive-prints (Feb. 2017).

[22] IL'IN, A., AND KHAS' MINSKII, R. On equations of Brownian motion. Theory of Probability \& Its Applications 9, 3 (1964), 421-444.

[23] KaVian, O., AND MischleR, S. The Fokker-Planck equation with subcritical confinement force. ArXiv e-prints (Dec. 2015).

[24] KaWAshima, S. The Boltzmann equation and thirteen moments. Japan J. Appl. Math. 7, 2 (1990), 301-320.

[25] Kolmogoroff, A. Zufällige Bewegungen (zur Theorie der Brownschen Bewegung). Ann. of Math. (2) 35, 1 (1934), 116-117.

[26] Mischler, S., AND Mounot, C. Exponential stability of slowly decaying solutions to the kinetic-Fokker-Planck equation. Arch. Ration. Mech. Anal. 221, 2 (2016), 677-723.

[27] Monmarché, P. A note on Fisher Information hypocoercive decay for the linear Boltzmann equation. ArXiv e-prints (Mar. 2017).

[28] Mounot, C., AND Neumann, L. Quantitative perturbative study of convergence to equilibrium for collisional kinetic models in the torus. Nonlinearity 19, 4 (2006), 969998.

[29] NASH, J. Continuity of solutions of parabolic and elliptic equations. Amer. J. Math. 80 (1958), 931-954. 
[30] Reed, M., AND Simon, B. Methods of modern mathematical physics. I, second ed. Academic Press, Inc. [Harcourt Brace Jovanovich, Publishers], New York, 1980. Functional analysis.

[31] VÁZQuez, J. L. Asymptotic behaviour methods for the Heat Equation. Convergence to the Gaussian. ArXiv e-prints (June 2017).

[32] Victory, JR., H. D., AND O'DwYer, B. P. On classical solutions of Vlasov-Poisson Fokker-Planck systems. Indiana Univ. Math. J. 39, 1 (1990), 105-156.

[33] Villani, C. Hypocoercive diffusion operators. In International Congress of Mathematicians. Vol. III. Eur. Math. Soc., Zürich, 2006, pp. 473-498.

[34] Villani, C. Hypocoercivity. Memoirs Amer. Math. Soc. 202, 2009.

October 5, 2017

E. BOUIN, CEREMADE (CNRS UMR n ${ }^{\circ}$ 7534), PSL research university, Université Paris-Dauphine, Place de Lattre de Tassigny, 75775 Paris 16, France $\bullet$ E-mail : bouin@ceremade.dauphine.fr

J. Dolbeault, CEREMADE (CNRS UMR n 7534), PSL research university, Université ParisDauphine, Place de Lattre de Tassigny, 75775 Paris 16, France E-mail: dolbeaul@ceremade.dauphine.fr

S. MischleR, CEREMADE (CNRS UMR n ${ }^{\circ} 7534$ ), PSL research university, Université ParisDauphine, Place de Lattre de Tassigny, 75775 Paris 16, France E-mail : mischler@ceremade.dauphine.fr

C. Моuнот, DPMMS, Center for Mathematical Sciences, University of Cambridge, Wilberforce Road, Cambridge CB3 0WA, UK • E-mail: C.Mouhot@dpmms.cam.ac.uk

C. SchmeIser, Fakultät für Mathematik, Universität Wien, Oskar-Morgenstern-Platz 1, 1090 Wien, Austria •E-mail:Christian.Schmeiser@univie.ac.at 